\title{
Quantitative Electrolysis of Droplet Contents in Microfluidic Channels. Concept and Experimental Validation.
}

Thomas Abadie, Christelle Souprayen, Catherine Sella and Laurent Thouin*

PASTEUR, Département de chimie, Ecole normale supérieure, PSL University, Sorbonne Université, CNRS, 75005, Paris, France

* Corresponding author:

laurent.thouin@ens.psl.eu

\begin{abstract}
A cross-channel geometry was studied to perform quantitative electrolysis of circulating droplets without influence of the counter electrode reaction on the droplet content. In the microdevices, the counter electrode is located in a secondary microchannel to prevent any contact with the droplets during detection. The electrochemical performance was assessed both theoretically and experimentally based on the droplet properties and mass transport above the working electrode. The results showed that under optimal conditions, quantitative electrolysis of droplet contents can be achieved when the working electrode operates in thin-layer regime. These results pave the way for new strategies allowing the implementation of electrochemical detections in droplet microfluidics without the need to perform calibration or to determine both the size and velocity of droplets prior to detection.
\end{abstract}

Keywords: microfluidics; droplet; microelectrode; amperometry; thin-layer regime. 


\section{INTRODUCTION}

Microfluidics has emerged as a distinct new field with a wide range of applications ranging from chemical synthesis to biological analysis. Its main interest originates from the development of microdevices implanting fluid flow at the microscale for high performance operations [1]. System miniaturization offers the ability to process small volumes of fluid with the control of chemical and biological entities both in time and space. All typical laboratory operations can be thus performed with significantly reduced volumes of samples / reagents, lower processing times and reaction times. Dropletbased microfluidics is one subdivision of microfluidics. It allows the generation and manipulation of drops contained within an immiscible carrier fluid [2-4]. In particular, immiscibility is leveraged to create discrete and small volumes moving within a continuous flow [5]. The benefits lie on the compartmentalization of chemical and biological reactions in droplets serving as individual microreactors or addressable reaction volumes [6]. A wide range of integrated and functional operations for droplet manipulation are available including generation, merging, sorting, splitting, storage and sampling [7]. Droplets can be processed individually and analyzed in parallel, enabling the efficient acquisition of large data sets for high-throughput screening and library building [8]. In recent years, droplet-based microfluidic tools have been used successfully in materials synthesis, single cell analysis [9-10], DNA/RNA sequencing and detection [11], small molecule screening [8], immunoassays [12] and tissue sampling [13], with great impact for enabling novel science [14].

The role of the quantitative analysis in the development of droplet-based systems is thus eminent and challenging. Despite the powerful detection methods used today, analytical techniques are still needed to improve detection performance and to expand the application fields [15]. Methods have been implemented like absorbance and fluorescence spectroscopy [16], laser-induced fluorescence [17], mass spectrometry [18], and Raman spectroscopy [19]. They provide selective, sensitive and accurate quantifications but their coupling with miniaturized systems for on-site applications remain unsuitable due to bulky and expensive instrumentations. In this context, electrochemical methods appear attractive and offer promising alternatives for implementing performant detections in microfluidic chips [20]. Microelectrodes can be easily integrated in microfluidic channels and allow very low volumes to be 
analyzed in real-time. However, only a few attempts have been addressed to detect electrochemically circulating droplets in microfluidic devices [21-23] or to probe their contents [24-31]. The applications were to analyze kinetics of reactions [24], to assay enzyme inhibition [26] or even to quantify biomolecules in the droplets $[29,31]$. Due to the discontinuous property of the two-phase flow, direct amperometric detection often leads to transient signals that are difficult to analyze [28]. In this case, the reliability of the measurements strongly depends on the breaking of the oil film around the droplets which initiates contact with the electrodes. To probe the droplet content, strategies have been proposed to overcome this limitation by locally converting the two-phase flow to a continuous flow before the detection step [32-35]. To achieve phase separation, the design of more elaborate channel networks is necessary [35-36] and requires in turn an accurate control of flow. The simplest approach remains to locate microband electrodes on microchannel walls while processing the minimum droplet sizes to ensure adequate contact time with the electrodes. Under these conditions, amperometric detection of droplets having large aspect ratio is carried out successfully [28-29,31,34]. Steady-state currents are recorded depending on the internal recirculating convection within droplets [37]. They allow the droplet content to be evaluated when the droplet volume and droplet velocity are measured simultaneously. Recently, we demonstrated that specific electrochemical cell configurations enable such determinations from chronoamperometric responses [38-39]. This approach was successfully validated for the titration of droplets after active merging with titrating solutions [39].

In the present paper, we wanted to extend this approach by performing a controlled and accurate electrolysis of droplet content. The objectives were here twofold: (i) to evaluate the droplet content without performing calibration whatever the velocity and size of the droplets, and (ii) to quantitatively electrolyze the droplet content without any limitation of the counter electrode operation (CE) on the final composition. Indeed, CE may produce interfering species which ultimately affect the analysis carried out at the working electrode (WE) and influence the droplet content after electrolysis. The first objective can be achieved by establishing a thin-layer operating regime at WE [40]. In this case, WE must quantitatively probe the element of solution passing above its surface. The second objective can be envisaged by implementing a junction between two microchannels in a specific channel network. Such cross-channel geometries also known as k-junction or k-channels, were originally created to 
develop in droplet microfluidics robust operations like reagent injection, fluid extraction, droplet splitting, and droplet delivering [41-46]. In this investigation, we wanted to design a novel electrochemical cell configuration based on these cross-channel geometries in order to exclude the operation of CE from the droplet electrolysis at WE. The principle is to locate WE in the main microchannel where droplets circulate, whereas inserting $\mathrm{CE}$ and the reference electrode (RE) in a secondary channel where an aqueous electrolyte flows continuously (Fig. 1). As a result, the junction between channels ensures the electrical contact between droplet and electrodes during the electrochemical detection. In the following, we investigated the operation of this new cell configuration and assessed its performance for a wide range of conditions. The influence of key parameters including channel geometries, electrode size, droplet size and droplet velocity was evaluated by numerical simulations. The data were compared to experimental measurements in order to test the predictions on the one hand, and to establish the proof of concept with underlying operating conditions on the other hand.

\section{EXPERIMENTAL}

The microfabrication processes of the microdevices were reported previously [37]. They consisted of classical hybrid glass chips with polydimethylsiloxane (PDMS) blocks. The networks comprising the two microchannels were engraved in a PDMS blocks whereas platinum electrodes as well as platinum optical markers were patterned on a glass slide by soft-lithography using lift-off procedures. The PDMS block was aligned on the glass slide with the help of two optical markers indicating the location of the junction on the patterns. The glass slide was irreversibly bonded with the PDMS block after plasma treatment.

The design of microdevices including the electrochemical cell configuration was based on a k-junction separating two microchannels having their own inlets and outlets. (Fig. 1). The main microchannel comprised upstream a flow-focusing geometry for on-demand droplet generation. The height $h$ of the microchannels was between 36 and $37.2 \mu \mathrm{m}$, depending on the molds used for casting the PDMS block. The width of the main microchannel was $200 \mu \mathrm{m}$ whereas the one of the secondary microchannel was 
$100 \mu \mathrm{m}$. In the main microchannel, the distance between the flow focusing geometry and the junction was about $1.5 \mathrm{~cm}$. The geometry of the junction is characterized by an intersection of $100 \mu \mathrm{m}$ between the two microchannels with an angle of $30^{\circ}$. The electrochemical cell was based on a three-electrode configuration. The working electrode (WE) was located in the main microchannel, at about $150 \mu \mathrm{m}$ downstream of the junction. The pseudo reference electrode (RE) and the counter electrode (CE) were both located in the secondary microchannel, upstream and downstream of the junction respectively. In order to ensure a good bonding between the PDMS block and the glass slide around the electrodes, and thus to avoid any liquid leakage, the RE and $\mathrm{CE}$ each consisted of 2 and 9 microbands $(160 \mu \mathrm{m}$ wide with gap distances of $60 \mu \mathrm{m}$ ) sharing a same conduction path, respectively. In contrast, WE consisted of a unique band placed along the main microchannel with several conducting paths. Between all the microdevices, only the WE size varied with $w_{\mathrm{WE}}=2000,700,500$ or $300 \mu \mathrm{m}$.

A silicon oil 150 AP Wacker (Sigma-Aldrich) was used as continuous organic phase and introduced in inlet 1. Aqueous solutions were introduced in the central inlet of the main microchannel (inlet 2) as the dispersed phase and in the inlet of the secondary microchannel (inlet3) as a continuous phase. For aqueous solutions, potassium chloride $(\mathrm{KCl}$, Acros Organics) was used as the supporting electrolyte at a minimal concentration of $0.5 \mathrm{~mol} \mathrm{~L}^{-1}$ to prevent any influence of ohmic drop. $210^{-3} \mathrm{~mol} \mathrm{~L}^{-1}$ potassium ferrocyanide $\left(\mathrm{K}_{4} \mathrm{Fe}(\mathrm{CN})_{6}\right.$, Acros Organics) in $\mathrm{KCl}$ solution was introduced at inlet 2 whereas a $\mathrm{KCl}$ solution was introduced at inlet 3 . The diffusion coefficient $D$ of ferrocyanide anion was equal to $810^{-}$ ${ }^{6} \mathrm{~cm}^{2} \mathrm{~s}^{-1}$. A pressure controller (MFCS-FLEX, Fluigent) controlled the injections of oil and aqueous solutions in all inlets. Each droplet was generated on demand by applying a pressure pulse over the aqueous solution in inlet 2 . The pressure at inlet 3 was tuned to ensure a constant flow in the secondary channel while maintaining a stable liquid-liquid interface at the junction and minimizing the transfer of solution towards the droplet after merging.

To optically control the generation of the droplets and to evaluate their characteristics along the main microchannel, the microdevices were placed under an inverted microscope (Axiovert 135, Zeiss) equipped with a CCD camera (AxioCam Icc1, Zeiss). After the droplet generation, and before the junction, their size $w_{0}$ and velocity $v_{0}$ were assessed using two optical markers separated by a gap 
distance of $1000 \mu \mathrm{m}$. Chronoamperometric measurements were carried out using a potentiostat (Autolab type PGSTAT30, Metrohm) driven by NOVA software. The working electrodes were biased at 0.4 $\mathrm{V} / \mathrm{RE}$ on the oxidation plateau of the ferrocyanide anion. Before each measurement, the electrodes were activated by performing in the aqueous phase several cyclic potential scans from -0.8 to $1.1 \mathrm{~V} / \mathrm{RE}$, at a potential scan rate of $0.7 \mathrm{Vs}^{-1}$.

Numerical simulations were performed in a 2D space using COMSOL Multiphysics software. Since the droplets had plug shape with high aspect ratio, the formulation of the problem was identical to those described in previous works considering a continuous flow of aqueous phase [40,47-49]. As for laminar flow, a parabolic flow profile was considered across the microchannel section due to the internal recirculating convection within the droplets. Assuming that the electrochemical reaction was only limited by mass transport, the diffusion-convection equation was solved numerically by finite elements with appropriate boundary conditions. The duration of electrode polarization corresponded to the contact duration between droplets and electrode.

\section{RESULTS AND DISCUSSION}

\subsection{Principle}

The design of the microfluidic devices is described in Fig. 1 with two rectangular microchannels. The width of the main microchannel is $L$. Each device comprises one inlet for the carrying fluid (oil, inlet 1 ), and two inlets for the aqueous phases, i.e., one for the introduction of droplet content in the main microchannel (inlet 2) and one for the continuous flow in the secondary microchannel (inlet 3). A flow focusing geometry is incorporated upstream of the main microchannel to generate on-demand the droplets. Upstream of the junction, two optical markers located in the main channel allow the determination of droplet characteristics, such as their duration of passage $\Delta t_{0}$, velocity $v_{0}$, and size $w_{0}$ according to a procedure already described [38]. The junction between the two microchannels allows a narrow contact between the two fluids while minimizing their mixing. The pseudo-reference electrode $\mathrm{RE}$ is placed in the secondary microchannel, upstream of the junction, to keep a constant reference 
potential without any influence of $\mathrm{CE}$, while $\mathrm{CE}$ is located downstream in the same microchannel. WE is placed in the main microchannel, downstream of the junction. According to this configuration, all the sequences taking place during the passage of the droplet in the main microchannel can be summarized and depicted in Fig. 2: (i) Before the droplet reaches the junction, the time $\Delta t_{0}$ can be monitored using one optical marker. (ii) Before any contact with WE, the droplet passes the junction and merges at the interface with the fluid of the secondary microchannel. (iii) The droplet reaches WE and connects the electrochemical cell, all the electrodes being in contact with the aqueous phase. (iv) As long as the electrochemical cell is connected with fluids in contact, the droplet is electrolyzed and a current response is monitored. (v) The upstream tail of the droplet elongates and narrows until it separates from the junction by disconnecting the electrochemical cell. Hence, during the time $\Delta t$ the electrochemical cell operates, the entire droplet is in contact with WE, but not with CE. According to this specific design, the influence of $\mathrm{CE}$ on the droplet content is prevented, allowing the control of a partial or total electrolysis of droplet content. Note that this principle remains valid as long as the droplet has a large aspect ratio in the main microchannel, i.e., with a size $w_{0}$ that remains greater than the microchannel width $L$.

An example of experimental response of such microdevices is given in Fig. 3A, showing the operation of the electrochemical cell during the passage of a droplet near the junction. When the electrical circuit is established, the current rises sharply and then decreases slowly until the disconnection. At the beginning, small spikes can be monitored probably due to the complex hydrodynamics initiated locally at the junction after the merging of the two fluids. Under these conditions, the detected charge $Q_{\mathrm{d}}$ during $\Delta t$ corresponds to the surface area of the chronoamperometric curve obtained by current integration.

\subsection{Theory}

The current response of the device can be simulated by only considering the mass transport above WE. Indeed, once the fusion is established at the junction between the two microchannels, the situation remains at first glance similar to the case of continuous flow for usual microchannel electrode configurations. The flow velocity profile within the droplets is parabolic due to internal recirculating convection and the corresponding average velocity can be approximated to the velocity of the circulating 
droplet. As in continuous flow, the electrode probes within the droplet a thin layer of fluid above its surface, whose characteristics are controlled locally by the flow velocity and geometries of both the microchannel and electrode. In this case, the formulation of the problem is the one already reported [40]. Since the width of the microchannel is larger than its height, the device can be described by a twodimensional system with one working electrode. To encompass all experimental conditions, the calculations were performed by introducing dimensionless parameters:

- coordinates

$$
X=\frac{x}{h} \text { and } Y=\frac{y}{h}
$$

- Electrode size

$$
W_{\mathrm{WE}}=\frac{w_{\mathrm{WE}}}{h}
$$

- Droplet size

$$
W=\frac{w}{h}
$$

- $\quad$ Time

$$
\tau=\frac{D t}{h^{2}}
$$

- Velocity with Peclet number

$$
P e=\frac{u_{\mathrm{av}} h}{D}
$$

- Current for one-electron transfer

$$
\psi=\frac{i}{\mathrm{~F} L D c^{0}}
$$

- Charge quantity

$$
\Theta=\frac{Q}{\mathrm{~F} L h^{2} c^{0}}
$$

with $h$ the microchannel height, $L$ the microchannel width, $w_{\mathrm{WE}}$ the electrode size, $u_{\mathrm{av}}$, the average flow velocity, $D$ the diffusion coefficient of the redox species, $c^{0}$ the concentration, $Q$ the electrical charge corresponding to the droplet content and F the Faraday.

One example of current response is displayed in Fig. 3B. The current increases theoretically with time up to a steady-state limiting value $\Psi_{\text {s }}$. This theoretical response is almost comparable to the experimental ones (Fig. 3A). The main difference is observed experimentally at short times before the regime reaches a steady state. As mentioned previously with the observation of current spikes, mass transport at the electrode results initially from the fusion process at the junction between the fluids. Once the steady state is reached, a plateau of current is almost observed as predicted theoretically. 
From a theoretical point of view; provided that the electrode size is large enough, a thin-layer regime can be established where the incoming content through the overall channel section is probed or detected by the electrode. The limiting current $\Psi_{\mathrm{s}}$ is thus given by a simple relation:

$\Psi_{\mathrm{s}}=P e$

This situation is illustrated in Fig. 3C for corresponding concentration profiles established within the microchannel during the progression of the droplet content above WE. Indeed, the species arriving at the electrode location can be totally electrolyzed for specific geometry $W_{\mathrm{WE}}$ and flow velocity $P e$. In this investigation, the complete electrochemical conversion of droplet content is the property on which the concept is presently based. Therefore, optimal conditions have to be delineated from simulations by considering the key parameters $W_{\mathrm{WE}}$ and $P e$. First, the minimal size $W_{\mathrm{s}}$ of droplet was evaluated according to the time $\tau_{\mathrm{s}}$ required to achieve steady-state regimes at WE. Indeed, a straightforward relation is foreseen with:

$W_{\mathrm{s}}=\tau_{\mathrm{s}} P e$

To this end, $\tau_{\mathrm{s}}$ was defined as the time needed to attain $0.95 \Psi_{\mathrm{s}}$. It was evaluated from simulated chronoamperometric curves (Fig. 3B). As evidenced in a seminal work, operating regimes of WE are imposed by the ratio $W_{\mathrm{WE}} / P e$. When $W_{\mathrm{WE}} / P e<1.2$, convective regimes including Levich regime take place. The electrode partially probes the incoming species at its upstream edge. In contrast, when $W_{\mathrm{WE}} / P e>1.2$, diffusion prevails over convection and the thin-layer regime is established. Hence, Fig. 4A shows the variation of $\tau_{\mathrm{s}}$ as a function of $W_{\mathrm{WE}} / P e$. Two distinct behaviors or domains can be observed. For $W_{\mathrm{WE}} / P e<1.2, \tau_{\mathrm{s}}$ almost equals to $W_{\mathrm{WE}} / P e$ whatever $W_{\mathrm{WE}}$ and $P e$ (see dashed curve in Fig. 4A). When $W_{\mathrm{WE}} / P e>1.2$, values of $\tau_{\mathrm{s}}$ are higher than 1.2 and are scattered within the corresponding domain. Under these conditions, $\tau_{\mathrm{s}}$ only depends on $P e$ and no longer on $W_{\mathrm{WE}}$ as shown in Fig. 4B by plotting $\tau_{\mathrm{s}}$ as a function of $P e$ for same data. Therefore, under thin-layer regime with $P e$ between 2 and $20, \tau_{\mathrm{s}}$ ranges between 1.2 and 2 . Since the maximal value of $\tau_{\mathrm{s}}$ is 2 , the minimal size of droplet $W_{\mathrm{s}}$ is thus: 
$W_{\mathrm{s}}=2 P e$

However, a second criterion about the droplet size can be foreseen by considering the charge quantity $\Theta_{\mathrm{d}}$ resulting from the integration of the chronoamperometric curve (Fig. 4C, gray area). Even though a thin-layer regime is established at the steady state, it appears that the droplet content cannot be fully probed by WE during the electrochemical cell connection $\Delta \tau$. A charge quantity $\Theta_{\text {nd }}$ remains undetermined due the time required for the regime to be established. This corresponds to the time needed for species to reach by diffusion the electrode surface from the top of the microchannel. This is clearly observed in Fig. 3C. At the time the cell is disconnected, a part of the droplet content has not been electrolyzed at its upstream edge as indicated by the presence of a concentration gradient. The resulting quantity is strictly equivalent to the complementary part $\Theta_{\text {nd }}$ of $\Theta_{\mathrm{d}}$ in Fig. 4C (white area). Thus, during the electrolysis of droplet content, the quantity $\Theta_{\mathrm{nd}}$ need to be minimized with respect to $\Theta_{\mathrm{d}}$. This amounts to considering a minimal size of droplet $W_{\min }$ greater than $W_{\mathrm{s}}$. In Fig. $4 \mathrm{D}$, the charge quantities $\Theta_{\mathrm{nd}}$ were first determined from simulations for different values of $W_{\mathrm{WE}}$ and $P e$. The data related to the thin-layer regime showed that $\Theta_{\mathrm{nd}}$ was approximately given by the relation (see linear curve fit in Fig. 4D):

$\Theta_{\text {nd }}=0.4 P e$

By introducing a threshold value such as:

$\frac{\Theta_{\text {nd }}}{\Theta_{\text {nd }}+\Theta_{\mathrm{d}}}=0.1$

it led to a corresponding droplet size $W_{\min }$ equals to:

$W_{\min }=4 P e$

Therefore, as expected in thin-layer regime, $W_{\min }$ depends only on the average flow velocity $P e$ but not on $W_{\text {WE}}$.

From what precedes, the following conclusions can be drawn: 
- When $W<2 P e$, the steady-state regime is not reached and the evaluation of the droplet content from electrolysis is not possible.

- When $2 P e<W<4 P e$, the thin-layer regime is established but the quantity $\Theta_{\text {nd }}$ cannot be minimized without considering the droplet velocity $P e$.

- $\quad$ When $W>4 P e$, the droplet content can be evaluated from $\Theta_{\mathrm{d}}$ without knowing its velocity $P e$.

\subsection{Experimental results}

Series of experiments were carried out using several microdevices with the same configuration previously described in Fig. 1. In each set, several droplets were generated on-demand by varying their size $w_{0}$ and velocity $v_{0}$ for microdevices with four electrode sizes $w_{\mathrm{WE}}$ (Table 1 ). After generation, the characteristics of the droplets $w_{0}, v_{0}$ and $\Delta t_{0}$ were determined using the optical markers located upstream of the junction (see experimental section).

In Fig. 5 are compared the durations $\Delta t$ of the chronoamperometric responses with the time $\Delta t_{0}$ of the droplets according to the electrode sizes $w_{\mathrm{WE}}$ investigated. In each case, linear variations were obtained with slopes close to unity. These results show that within the experimental uncertainties, the electrochemical responses were consistent with the initial characteristic $\Delta t_{0}$ of the droplets. No alterations induced by their passage at the junction and during their detection at WE were experimentally observed. These results validate at first glance the expected operation of the microfluidic circuit. Note nevertheless that slopes of curve fits were slightly lower than 1. It could be explained by a slight increase of the droplet velocity $v$ downstream of the junction (i.e., lower $\Delta t$ ) initiated by a local pressure drop inside the main microchannel after merging. In parallel, it could also be due to a delay in the rupture of the oil films surrounding the droplet on the electrode surface. In such a case, it depends on the wettability of the electrodes from one measurement to the other. For all data, deviations were evaluated within 5\%.

In Fig. 6 are plotted the average currents $i_{\mathrm{av}}$ evaluated from the chronoamperometric curves (see in Fig. 3A) as a function of the droplet velocity $v_{0}$. As expected, $i_{\text {av }}$ increases linearly with $v_{0}$ for the largest 
electrode $w_{\mathrm{WE}}=2000 \mu \mathrm{m}$. In this case, the thin-layer is well established and the current follows Eq. (1) when $v_{0}=u_{\mathrm{av}}$ :

$i_{\mathrm{av}}=\mathrm{F} L h v_{0} c^{0}$

The theoretical condition $W_{\mathrm{WE}} / \mathrm{Pe}>1.2$ was checked for all the data. A good agreement was observed between experimental (symbols) and theoretical (solid line) currents, thus validating the prediction for thin-layer regime. When $w_{\mathrm{WE}}=700 \mu \mathrm{m}$, experimental currents also followed Eq. (7) (solid line) but only for velocities $v_{0}$ lower than $200 \mu \mathrm{m} \mathrm{s}^{-1}$. Under these conditions, deviations were indeed predicted as shown in Fig. 6 by the corresponding variation of simulated current (dashed line). This behavior is linked to the transition between thin-layer regime and intermediate regimes which are promoted by convection. Note that experimentally, deviations from theory were observed at the highest velocities $v_{0}$. This can be explained by a lower active surface area of WE due to electrode wettability. This situation is obviously encountered for $w_{\mathrm{WE}}=500 \mu \mathrm{m}$ and $w_{\mathrm{WE}}=300 \mu \mathrm{m}$ with deviations taking place from velocities about 150 to $200 \mu \mathrm{m} \mathrm{s}^{-1}$. Therefore, for all electrode sizes, the same experimental trends were observed, validating within the experimental uncertainties the predictions derived under thin-layer regime The good agreement observed at low droplet velocities between Eq. (7) and experimental data show that after the junction the droplet velocity did not change drastically. This means in turn that no drastic mixing between the two microchannels took place after merging. Moreover, it must be highlighted that under these specific conditions, data are no longer influenced by the active surface area of the electrodes. Indeed, the electrodes are large enough to compensate any experimental artefact that may result from weak electrode activation or electrode wettability.

Charge quantities $Q_{\mathrm{d}}$ were thus evaluated from the same set of data, by integrating the chronoamperometric curves, while clearly distinguishing the ones corresponding to the thin-layer regime. Values of $Q_{\mathrm{d}}$ were then compared in Fig. 7 with the charge quantity $Q_{0}$ of droplets estimated from the concentration $c_{0}$ and initial sizes $w_{0}$ determined optically. For data only associated to the thinlayer regime (solid symbols), linear variations were well observed. Slopes of linear curve fits (dashed lines) were close to 1 with values ranging between 0.94 and 0.97 . Data from the other regimes (open symbols) were not considered here since they fully diverged from $Q_{0}$ as it was predicted theoretically. 
Nevertheless, despite the thin-layer regime was established, the electrolysis of droplets from slopes was not quantitative, whatever the electrode size $w_{\mathrm{WE}}$. One reason is probably the contribution of the undetected charge quantity $Q_{\text {nd }}$ on the experimental charge determination. By considering Eq. (4) established previously with dimensionless parameters, $Q_{\text {nd }}$ can now be estimated by:

$Q_{\mathrm{nd}}=0.4 \frac{\mathrm{F} L h^{3} v_{0} c^{0}}{D}$

$Q_{\text {nd }}$ was thus calculated from Eq. (8). Depending on the experimental conditions used, $Q_{\text {nd }}$ did not exceed $0.1 Q_{\mathrm{d}}$. Values of $Q_{\text {nd }}$ and the sum $\left(Q_{\mathrm{d}}+Q_{\text {nd }}\right)$ were plotted in Fig. 8 as a function of the droplet content $Q_{0}$ for $w_{\mathrm{WE}}=2000 \mu \mathrm{m}$. The slope of the linear curve fit deduced from the sum $\left(Q_{\mathrm{d}}+Q_{\mathrm{nd}}\right)$ was equal to 1.016 as compared to the one of 0.964 obtained from $Q_{\mathrm{d}}$ (Fig. 7). The charge quantity $Q_{\text {nd }}$ may thus

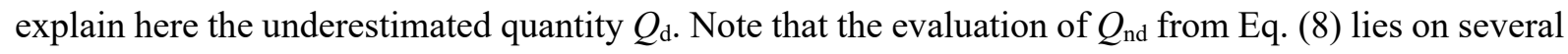
experimental parameters including the diffusion coefficient. As a result, introducing $Q_{\text {nd }}$ within the sum $\left(Q_{\mathrm{d}}+Q_{\text {nd }}\right)$, data in Fig. 8 leads to data which are more scattered (as compared to Fig. 7). As stated above, the accuracy in the determination of droplet content depends on a criterion based on the ratio W/Pe (see Eq. (6)). For $w_{\mathrm{WE}}=2000 \mu \mathrm{m}$, without considering $Q_{\mathrm{nd}}$ the droplet content was determined within 5\% accuracy for an average $\mathrm{W} / \mathrm{Pe}$ value close to 8 . Better accuracy can be envisaged by increasing the size of the droplets and /or by decreasing their velocity. Increasing the size of WE should also minimize to some extent experimental artefacts related to electrode wettability by ensuring a thin-layer regime under all conditions. Finally, the cross-channel geometry could be optimized according to the operating conditions (i.e., the intersection between the two microchannels, location of the upstream edge of WE versus the junction...). Nevertheless, optimizing the device was not under the scope of this study since the main objective was to successfully demonstrate the concept for quantitative electrolysis of droplets without influence of the counter electrode on their final composition. Note that when all the electrodes are located in a single microchannel, full conversion of droplet content remains impossible since a part of the droplet is never detected during the connection of the electrochemical cell, whatever the configuration (i.e., when $\mathrm{CE}$ is located upstream or downstream of WE). Therefore, without a cross- 
channel geometry, the electrolysis cannot be considered as a means of converting quantitatively the composition of droplets within a sequence of steps in a microfluidic circuit.

\section{CONCLUSION}

The cross-channel geometry was implemented to allow the quantitative electrolysis of droplets flowing in a microfluidic channel in a three-electrode cell configuration. In such microdevices, the influence of the counter electrode on the droplet content is eliminated since this electrode operates in a separate channel. The validity of the electrochemical cell has been theoretically evaluated and validated experimentally as a function of the droplet properties and operating regimes of the working electrode. Under these conditions, the sizes of droplet and sizes of working electrode have been delineated in order to ensure optimum electrochemical performance. Therefore, electrolysis of droplet content can be performed and controlled experimentally, in particular in thin-layer regime for quantitative evaluation or quantitative conversion of droplet content. Unlike conventional amperometric measurements, droplet velocity and droplet volume do not have to be preliminary determined. Furthermore, the size of the working electrode should only meet the minimum-size criterion according the experimental conditions used. Therefore, the configuration of this innovative electrochemical cell paves the way for the design in droplet microfluidics of versatile platforms implementing an electrochemical detection. This approach fills the gap in the electrochemical detection of droplets.

\section{CRediT authorship contribution statement}

Thomas Abadie: Methodology, Investigation. Christelle Souprayen: Investigation. Catherine Sella: Validation, Formal analysis. Laurent Thouin: Conceptualization, Validation, Formal analysis, Writing - Original Draft Review \& Editing, Supervision. 


\section{Declaration of competing interest}

The authors declare that they have no known competing financial interests or personal relationships that could have appeared to influence the work reported in this paper.

\section{Acknowledgements}

This work was supported in parts by CNRS UMR 8640, ENS (Ecole normale superieure), PSL University and Sorbonne Université. T.A. thanks the doctoral school ED388 for a PhD grant. 


\section{REFERENCES}

[1] G. M. Whitesides, Nature 442 (2006) 368.

[2] T. Thorsen, R. W. Roberts, F. H. Arnold, S. R. Quake, Phys. Rev. Lett. 86 (2001) 4163.

[3] H. Song, M. R. Bringer, J. D. Tice, C. J. Gerdts, R. F. Ismagilov, Appl. Phys. Lett. 83 (2003) 4664.

[4] T. M. Squires, S. R. Quake, Rev. Mod. Phys. 77 (2005) 977.

[5] P. Zhu, L. Wang, Lab Chip 17 (2017) 34.

[6] S. Y. Teh, R. Lin, L. H. Hung, A. P. Lee, Lab Chip 8 (2008) 198.

[7] L. Shang, Y. Cheng, Y. Zhao, Chem. Rev. 117 (2017) 7964.

[8] E. M. Payne, D. A. Holland-Moritz, S. Sun, R. T. Kennedy, Lab Chip 20 (2020) 2247.

[9] D. Gao, F. Jin, M. Zhou, Y. Jiang, The Analyst 144 (2019) 766.

[10] K. Matula, F. Rivello, W. T. S. Huck, Adv. Biosys. 4 (2020) e1900188.

[11] R. Williams, S. G. Peisajovich, O. J. Miller, S. Magdassi, D. S. Tawfik, A. D. Griffiths, Nat Methods 3 (2006) 545.

[12] R. Gao, Z. Cheng, A. J. deMello, J. Choo, Lab Chip 16 (2016) 1022.

[13] A. M. Nightingale, C. L. Leong, R. A. Burnish, S. U. Hassan, Y. Zhang, G. F. Clough, M. G. Boutelle, D. Voegeli, X. Niu, Nat Commun 10 (2019) 2741.

[14] A. Suea-Ngam, P. D. Howes, M. Srisa-Art, A. J. deMello, Chem. Commun. 55 (2019) 9895.

[15] W. W. Liu, Y. Zhu, Anal. Chim. Acta 1113 (2020) 66.

[16] T. Yang, S. Stavrakis, A. deMello, Anal. Chem. 89 (2017) 12880.

[17] F. Ma, M. T. Chung, Y. Yao, R. Nidetz, L. M. Lee, A. P. Liu, Y. Feng, K. Kurabayashi, G. Y. Yang, Nat Commun 9 (2018) 1030.

[18] T. Ngernsutivorakul, D. J. Steyer, A. C. Valenta, R. T. Kennedy, Anal. Chem. 90 (2018) 10943.

[19] G. L. Nelson, S. E. Asmussen, A. M. Lines, A. J. Casella, D. R. Bottenus, S. B. Clark, S. A. Bryan, Anal. Chem. 90 (2018) 8345.

[20] B. K. Kim, J. Kim, A. J. Bard, J. Am. Chem. Soc. 137 (2015) 2343. 
[21] S. Liu, Y. Gu, R. B. Le Roux, S. M. Matthews, D. Bratton, K. Yunus, A. C. Fisher, W. T. Huck, Lab Chip 8 (2008) 1937.

[22] E. V. Moiseeva, A. A. Fletcher, C. K. Harnett, Sens. Actuators, B 155 (2011) 408.

[23] Y. Gu, A. C. Fisher, The Analyst 138 (2013) 4448.

[24] Z. Han, W. Li, Y. Huang, B. Zheng, Anal. Chem. 81 (2009) 5840.

[25] Z. Han, Y. Y. Chang, S. W. Au, B. Zheng, Chem. Commun. 48 (2012) 1601.

[26] S. Gu, Y. Lu, Y. Ding, L. Li, F. Zhang, Q. Wu, Anal. Chim. Acta 796 (2013) 68.

[27] S. Gu, Y. Lu, Y. Ding, L. Li, H. Song, J. Wang, Q. Wu, Biosens. Bioelectron. 55 (2014) 106.

[28] X. Hu, X. Lin, Q. He, H. Chen, J. Electroanal. Chem. 726 (2014) 7.

[29] A. Suea-Ngam, P. Rattanarat, O. Chailapakul, M. Srisa-Art, Anal. Chim. Acta 883 (2015) 45.

[30] P. Rattanarat, A. Suea-Ngam, N. Ruecha, W. Siangproh, C. S. Henry, M. Srisa-Art, O. Chailapakul, Anal. Chim. Acta 925 (2016) 51.

[31] A. Suea-Ngam, P. Rattanarat, K. Wongravee, O. Chailapakul, M. Srisa-Art, Talanta 158 (2016) 134.

[32] F. Sassa, H. Laghzali, J. Fukuda, H. Suzuki, Anal. Chem. 82 (2010) 8725.

[33] L. A. Filla, D. C. Kirkpatrick, R. S. Martin, Anal. Chem. 83 (2011) 5996.

[34] H. Liu, R. M. Crooks, Lab Chip 13 (2013) 1364.

[35] X. Lin, X. Hu, Z. Bai, Q. He, H. Chen, Y. Yan, Z. Ding, Anal. Chim. Acta 828 (2014) 70.

[36] X. Niu, F. Pereira, J. B. Edel, A. J. de Mello, Anal. Chem. 85 (2013) 8654.

[37] T. Abadie, C. Sella, L. Thouin, Electrochem. Commun. 80 (2017) 55.

[38] T. Lombardo, L. Lancellotti, C. Souprayen, C. Sella, L. Thouin, Electroanalysis 31 (2019) 2103.

[39] T. Delahaye, T. Lombardo, C. Sella, L. Thouin, Anal. Chim. Acta 1155 (2021) 338344.

[40] C. Amatore, N. Da Mota, C. Sella, L. Thouin, Anal. Chem. 79 (2007) 8502.

[41] D. Chen, W. Du, Y. Liu, W. Liu, A. Kuznetsov, F. E. Mendez, L. H. Philipson, R. F. Ismagilov, Proc. Natl. Acad. Sci. USA 105 (2008) 16843.

[42] G. T. Roman, M. Wang, K. N. Shultz, C. Jennings, R. T. Kennedy, Anal. Chem. 80 (2008) 8231.

[43] R. Lin, J. S. Fisher, M. G. Simon, A. P. Lee, Biomicrofluidics 6 (2012) 24103.

[44] Y. Ding, X. Casadevall i Solvas, A. deMello, The Analyst 140 (2015) 414. 
[45] U. Tangen, A. Sharma, P. Wagler, J. S. McCaskill, Biomicrofluidics 9 (2015) 014119.

[46] S. R. Doonan, R. C. Bailey, Anal. Chem. 89 (2017) 4091.

[47] C. Amatore, C. Lemmer, P. Perrodin, C. Sella, L. Thouin, Electrochem. Commun. 13 (2011) 1459.

[48] C. Amatore, C. Lemmer, C. Sella, L. Thouin, Anal. Chem. 83 (2011) 4170.

[49] T. Abadie, C. Sella, P. Perrodin, L. Thouin, Front. Chem. 7 (2019) 704. 


\begin{tabular}{|c|c|c|}
\hline$w_{\mathrm{WE}} / \mu \mathrm{m}$ & $w_{0} / \mu \mathrm{m}$ & $v_{0} / \mu \mathrm{m} \mathrm{s}^{-1}$ \\
\hline 2000 & $1748<w_{0}<4456$ & $142<v_{0}<405$ \\
\hline 700 & $1601<w_{0}<3310$ & $65<v_{0}<309$ \\
\hline 500 & $1704<w_{0}<2729$ & $76<v_{0}<175$ \\
\hline 300 & $1610<w_{0}<2783$ & $69<v_{0}<247$ \\
\hline
\end{tabular}

Table 1. Properties $w_{0}$ and $v_{0}$ of the droplets investigated with four sizes $w_{\mathrm{WE}}$ of working electrode. 


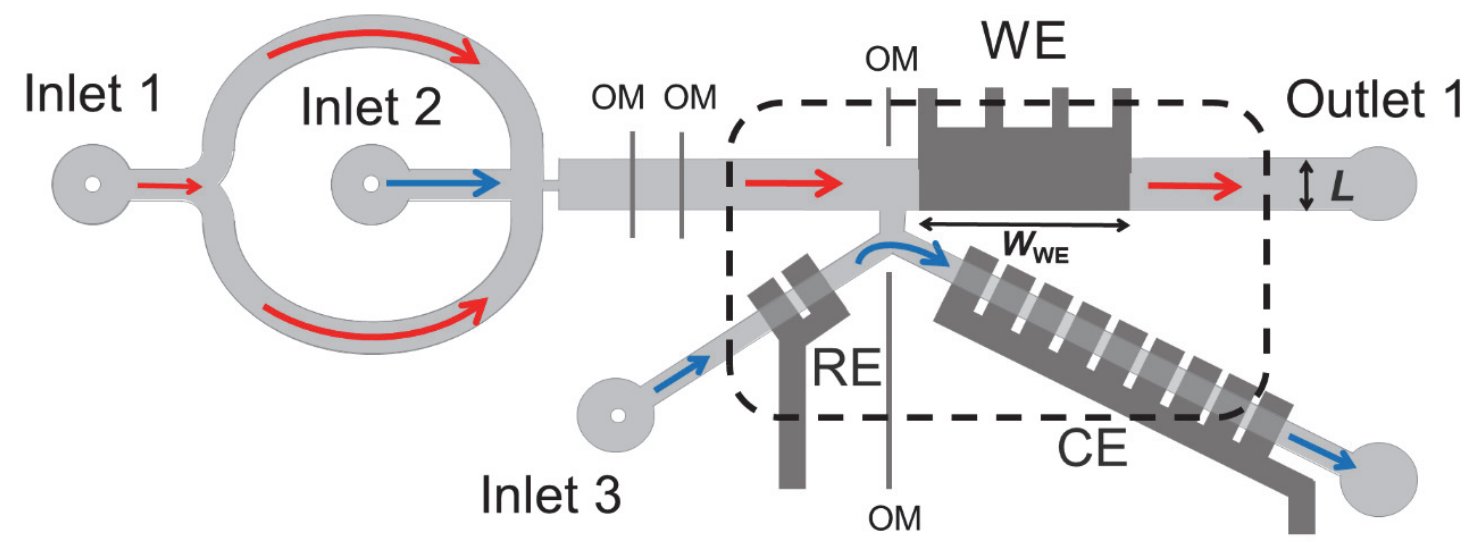

Outlet 2
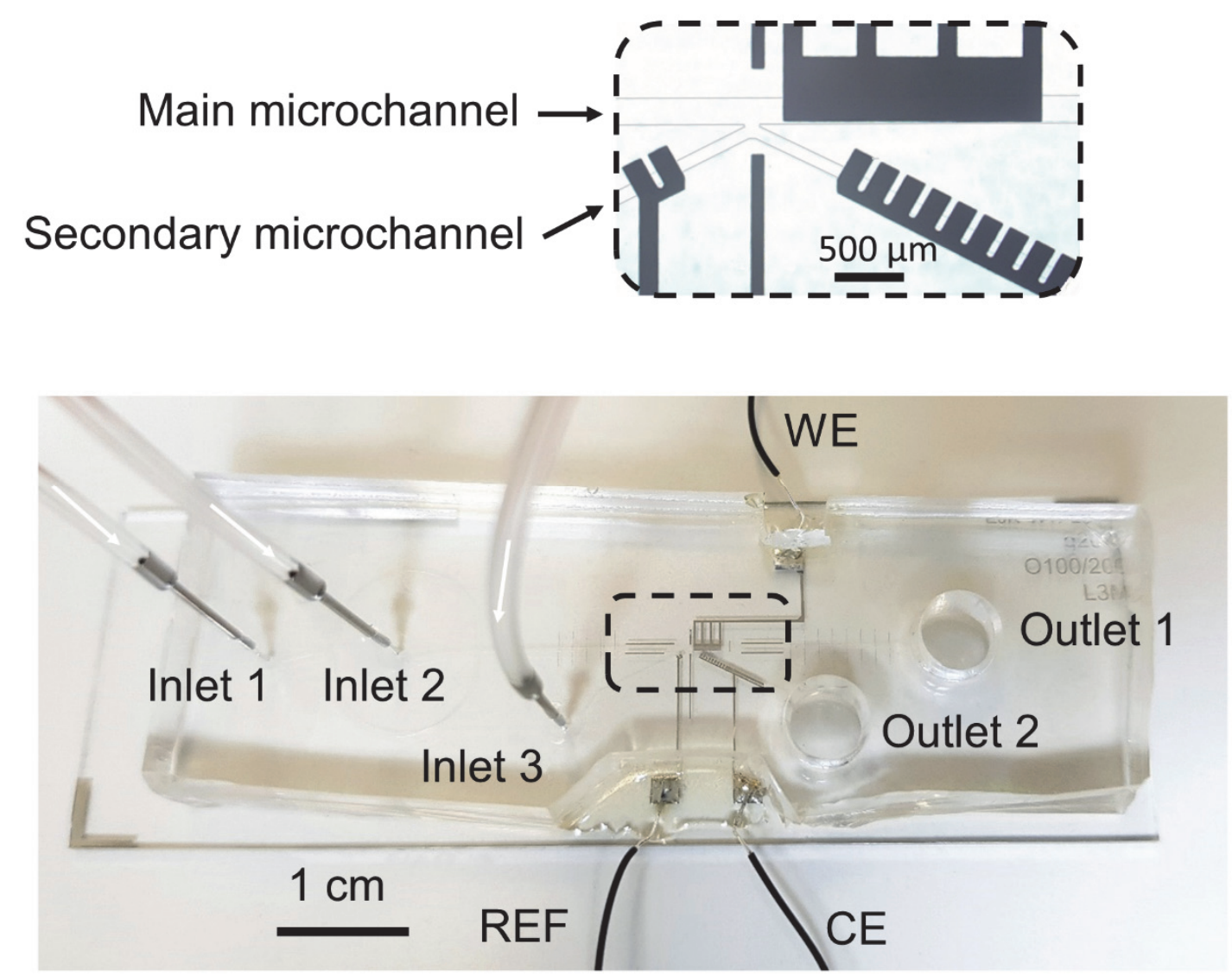

Fig. 1. Design of the microfluidic device that integrates upstream a flow-focusing geometry for ondemand droplet generation, a cross-channel geometry, and three electrodes with their connection paths. Two photos (middle and bottom) taken from the top of a device with $w_{\mathrm{WE}}=2000 \mu \mathrm{m}$ and $L=200 \mu \mathrm{m}$. The dashed line delineates the electrochemical cell with the working electrode (WE), reference electrode (RE) and counter electrode (CE). Optical markers (OM), carrying fluid (red arrows) and aqueous phases (blue arrows). 


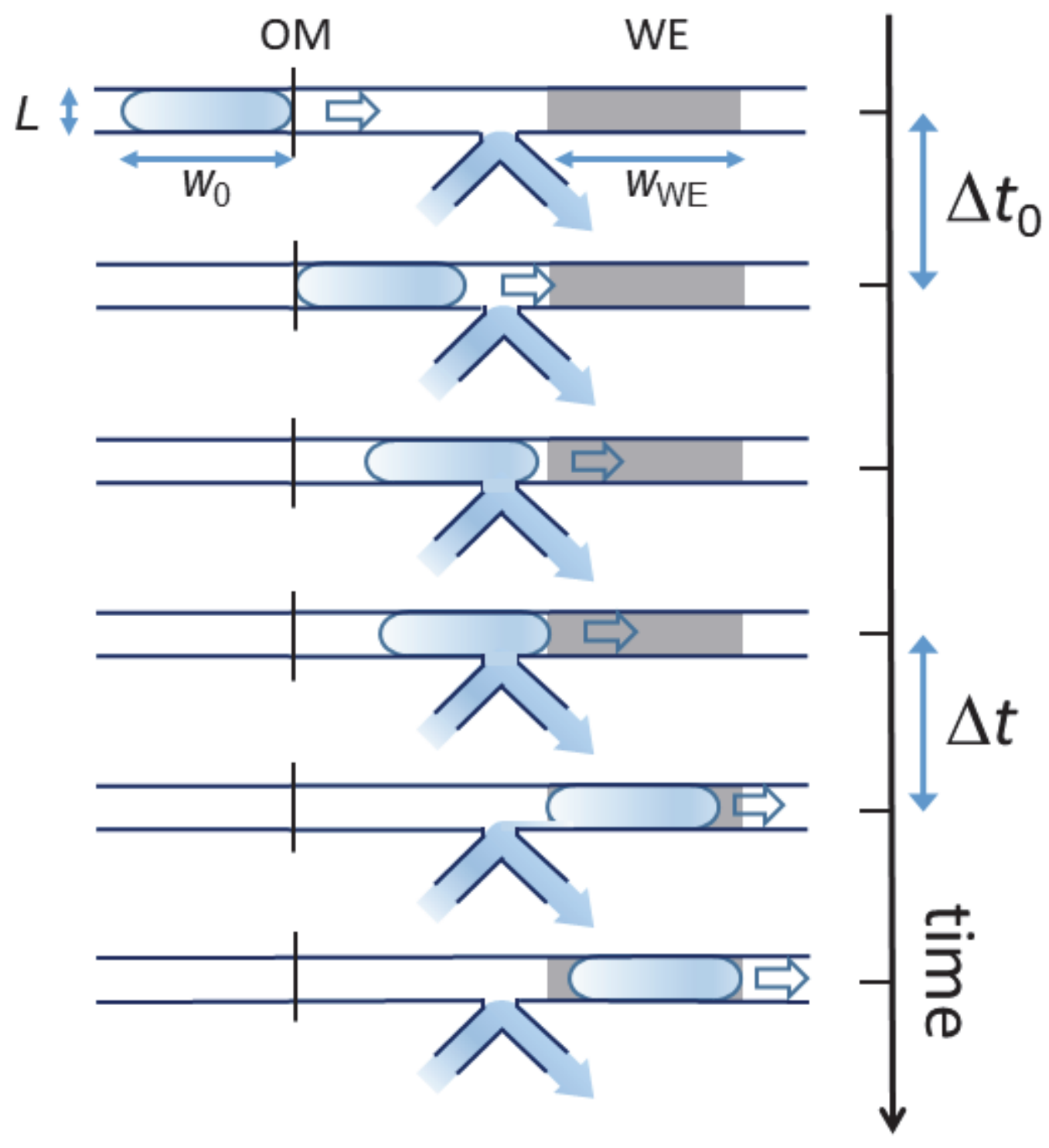

Fig. 2. Illustration in the main microchannel of the droplet detection versus time. $\Delta t_{0}$ is the duration of passage of the droplet over the optical marker (OM). $\Delta t$ is the time duration during which the electrochemical cell is connected. The reference and counter electrodes in the secondary microchannel are not represented. 
A)

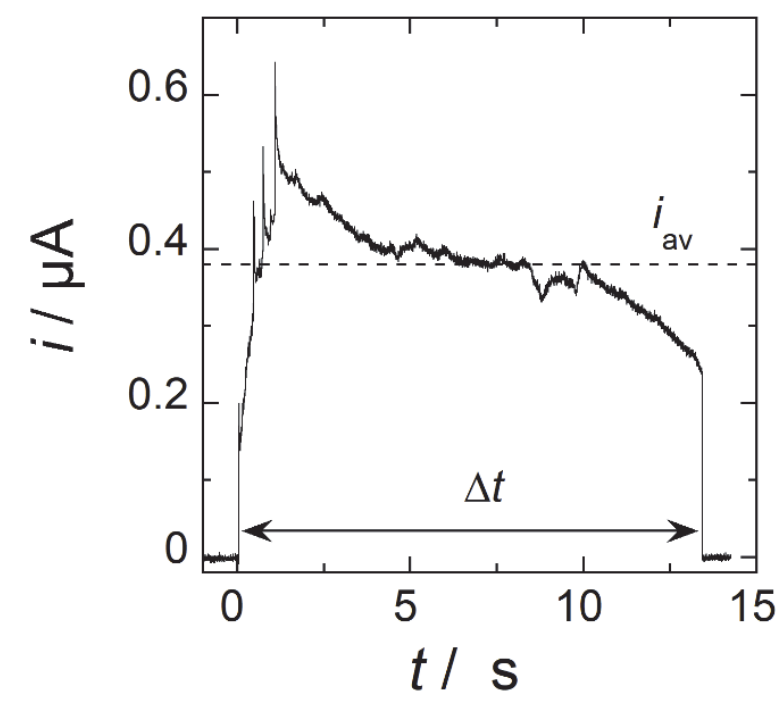

B)

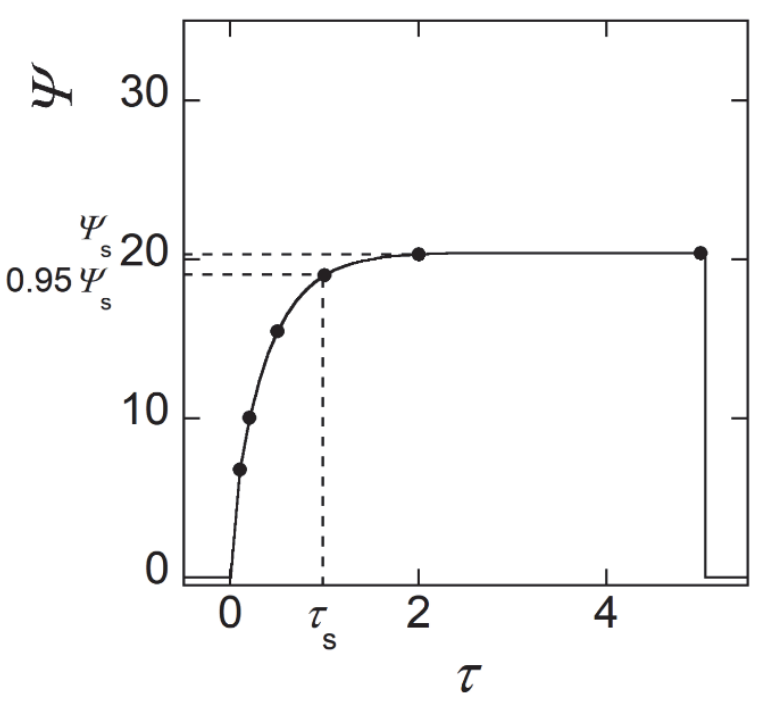

C)

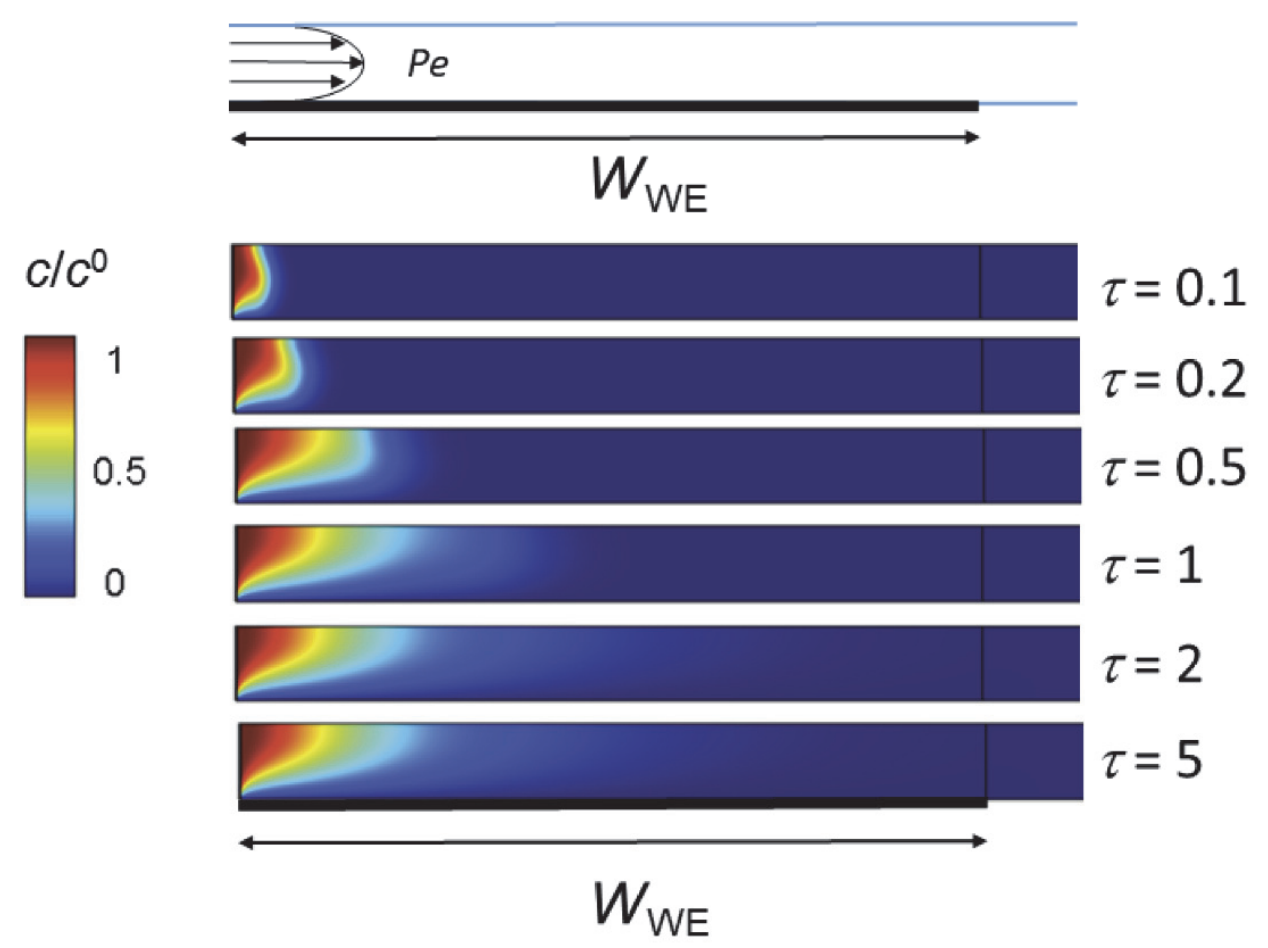

Fig. 3. A) Experimental response monitored for $w_{\mathrm{WE}}=2000 \mu \mathrm{m}, v=181 \mu \mathrm{m} \mathrm{s}^{-1}$, and $w_{0}=2696 \mu \mathrm{m}$. B) Theoretical response simulated in thin-layer regime for $W_{\mathrm{WE}}=50$ and $P e=20$ with $W_{0}=100$. Definitions of current $\Psi_{\mathrm{s}}$ and time $\tau_{\mathrm{s}}$. C) 2D-concentration profiles simulated within the microchannel above the working electrode at different times corresponding to symbols in B). On top, schematic representation of the velocity profile. 
A)

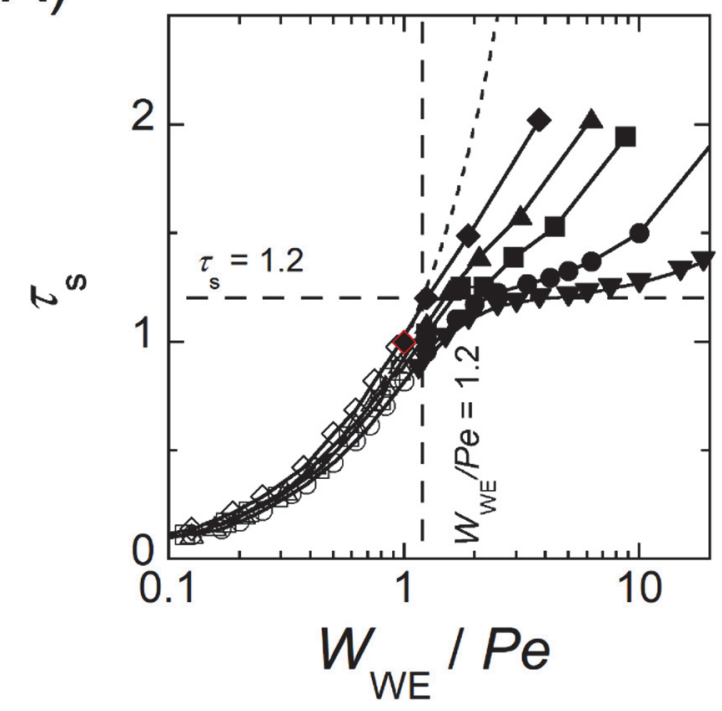

C)

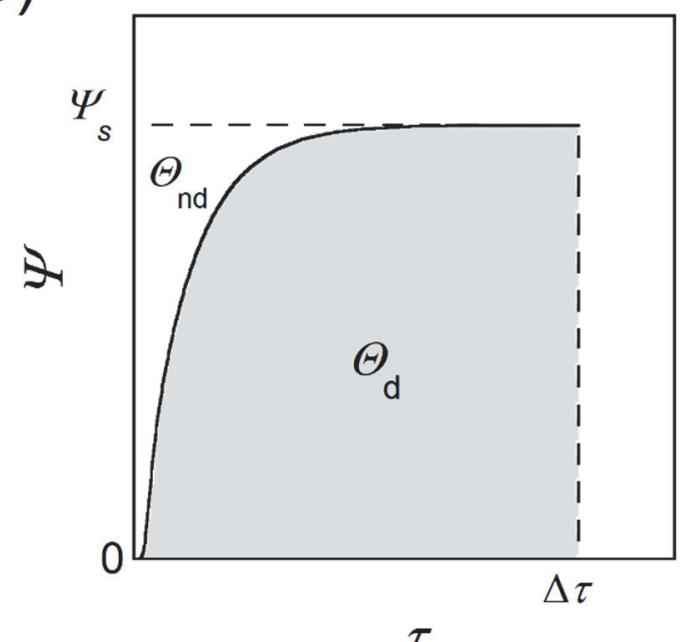

B)

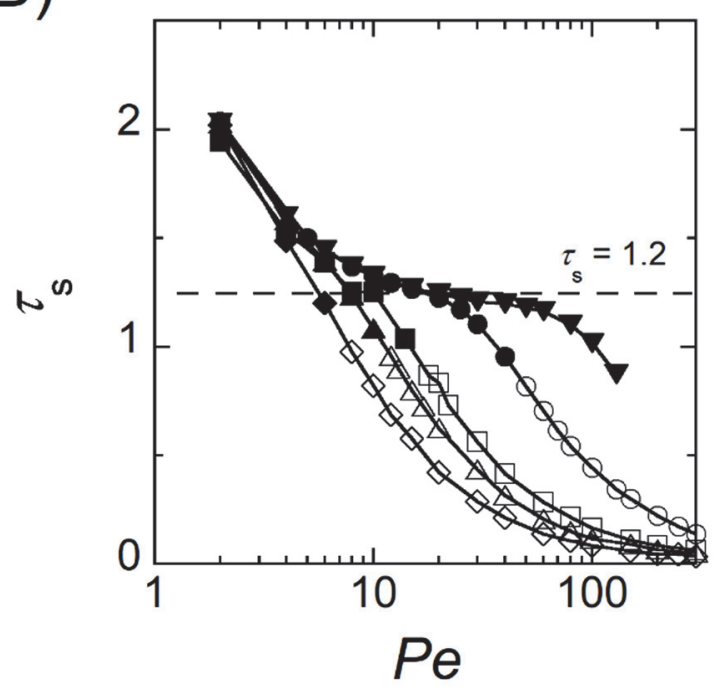

D)

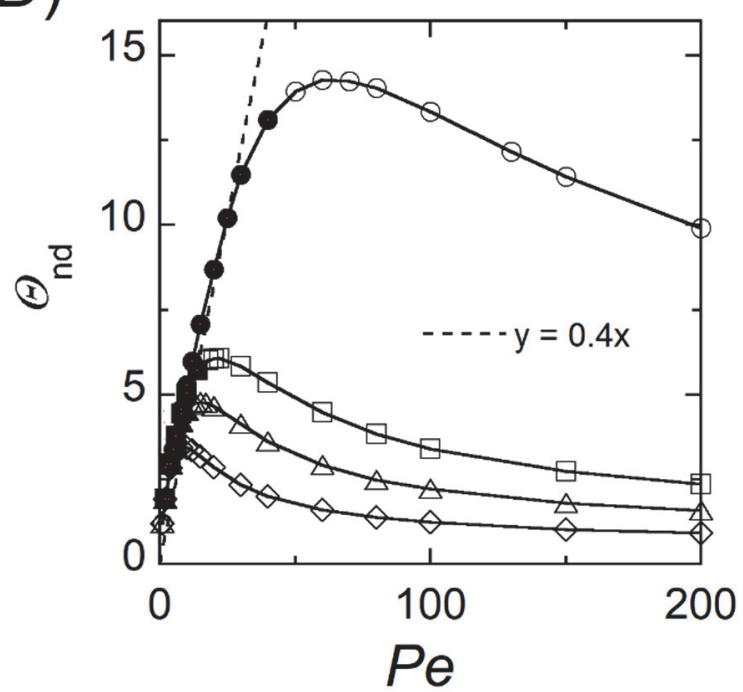

Fig. 4. A) Variation of $\tau_{\mathrm{s}}$ as a function of $W_{\mathrm{WE}} / P e$. B) Variation of $\tau_{\mathrm{s}}$ as a function of $P e$. C) Definition of the charge quantities $\Theta_{\mathrm{d}}$ and $\Theta_{\mathrm{nd}}$ from a theoretical current response. $\Theta_{\mathrm{d}}$ corresponds to the gray area whereas $\Theta_{\text {nd }}$ corresponds to the complementary white area. $\Delta \tau$ is the time duration during which the electrochemical cell is connected. D) Variation of $\Theta_{\mathrm{nd}}$ as a function of $P e$. In (A-B,D) $W_{\mathrm{WE}}=7.5$ (diamond), 12.5 (triangle) 17.5 (square), 50 (circle) and 150 (inverted triangle). Solid symbols are for $W_{\mathrm{WE}} / P e>1.2$ whereas open symbols are for $W_{\mathrm{WE}} / P e<1.2$. 

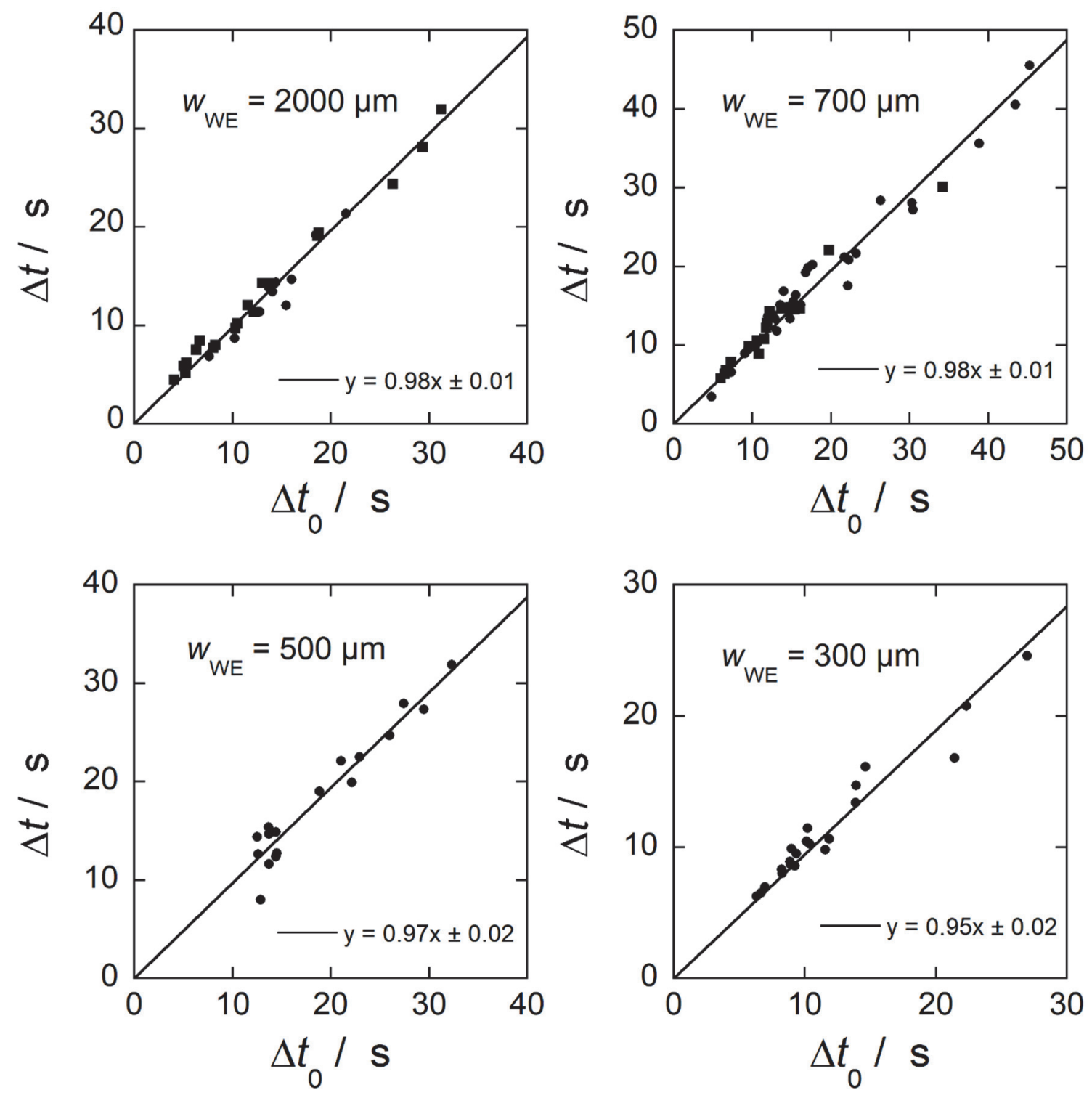

Fig. 5. Comparison between the time duration $\Delta t$ of droplet detection and $\Delta t_{0}$ of droplets for different sizes $w_{\mathrm{WE}}$ of working electrodes. $\Delta t_{0}=w_{0} / v_{0}$. 

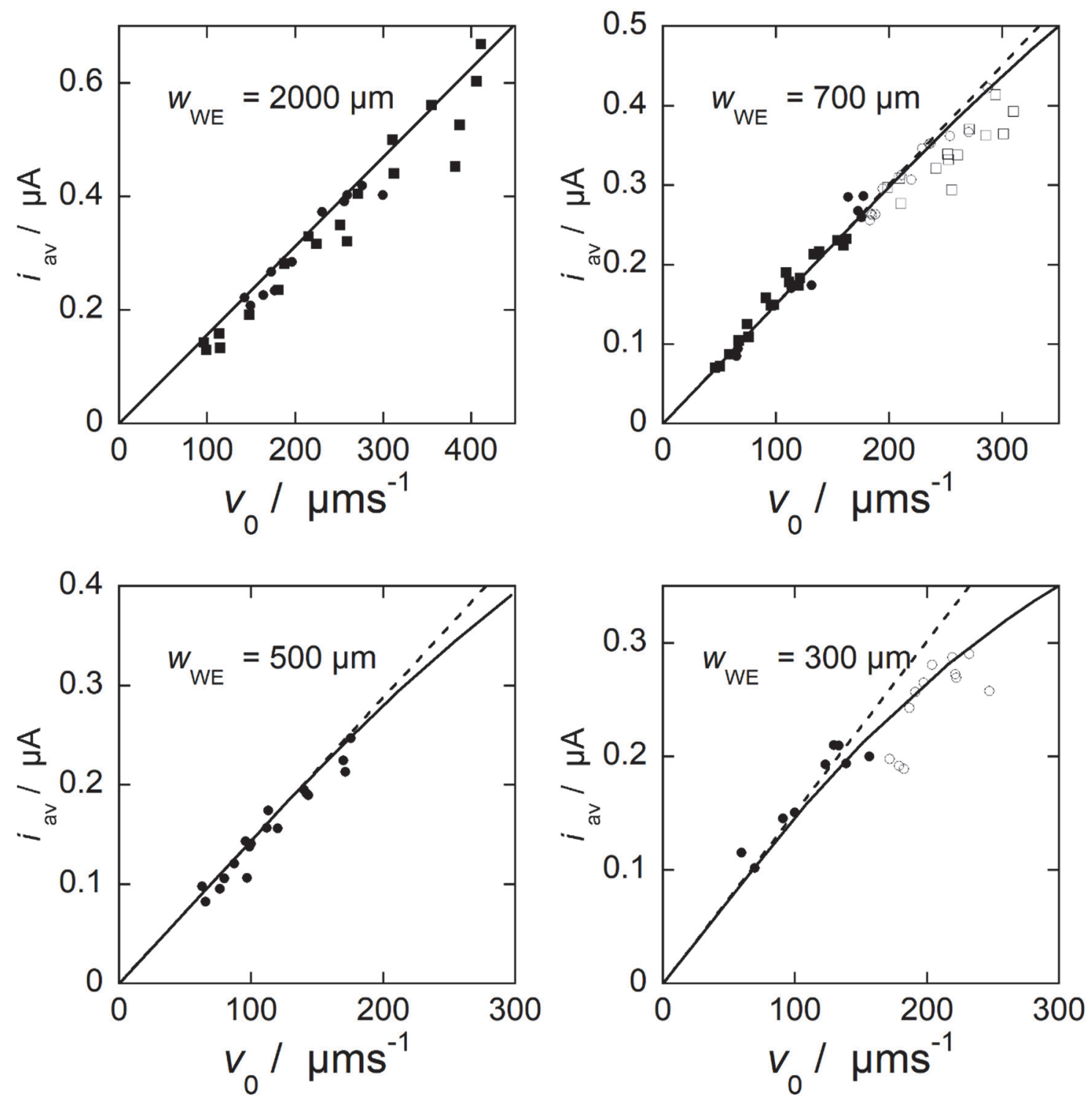

Fig. 6. Comparison between experimental (symbols) and theoretical (curves) average current $i_{\text {av }}$ as a function of the droplet velocity $v_{0}$ for different sizes $w_{\mathrm{WE}}$ of working electrodes: Theoretical currents comprising all regimes (solid lines), theoretical currents for thin-layer regime only (dashed lines), experimental current corresponding to thin-layer regime (solid symbols), and experimental currents corresponding to transition regimes (open symbols). 

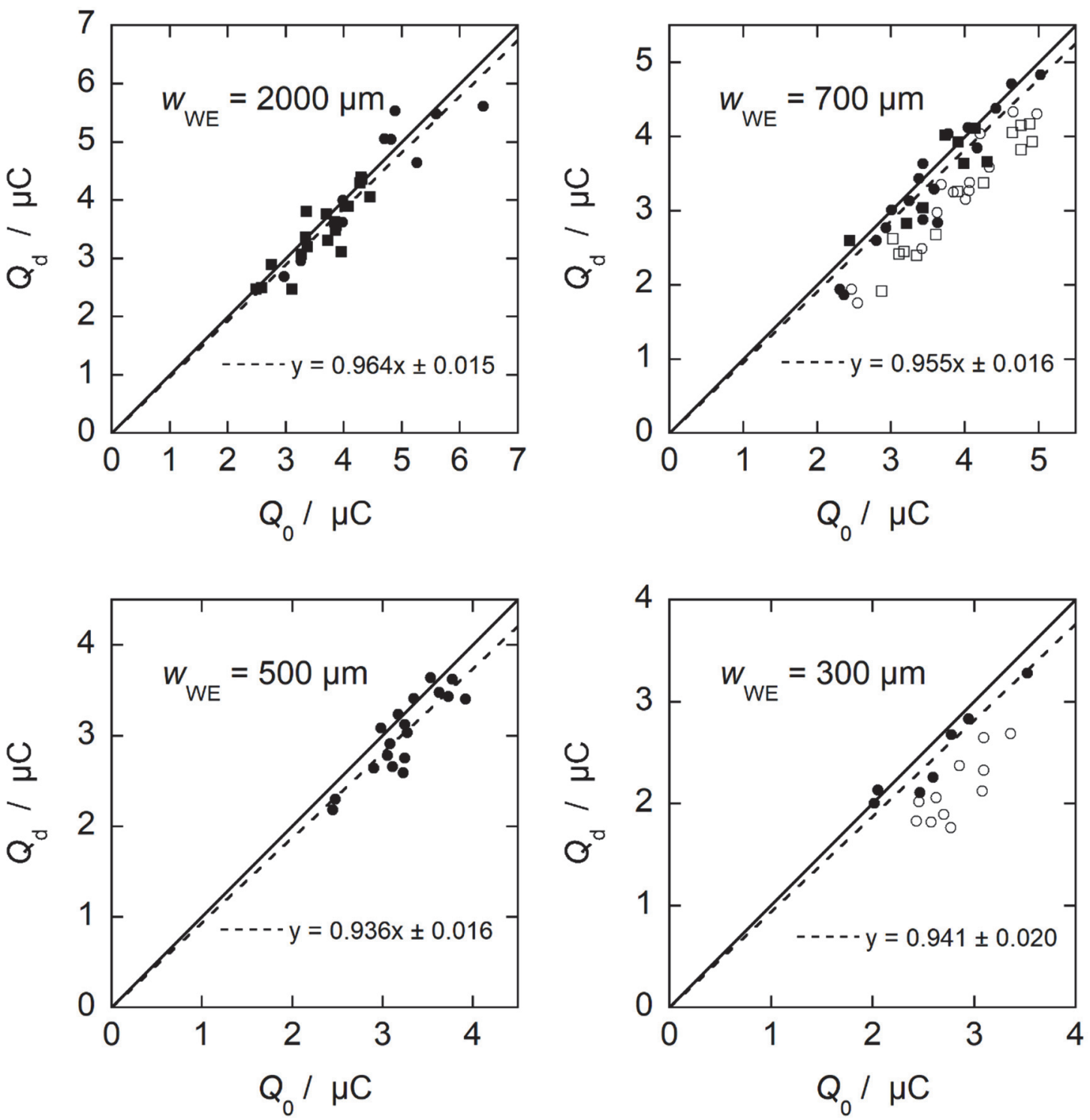

Fig. 7. Comparison between experimental (symbols) and theoretical (curves) charge quantities $Q_{\mathrm{d}}$ as a function of the droplet charge $Q_{0}$ for different sizes $w_{\mathrm{WE}}$ of working electrodes. Theoretical charges (solid lines), linear curve fits (dashed lines) of $Q_{\mathrm{d}}$ (solid symbols) in thin-layer regime, and $Q_{\mathrm{d}}$ (open symbols) corresponding to transition regimes. 


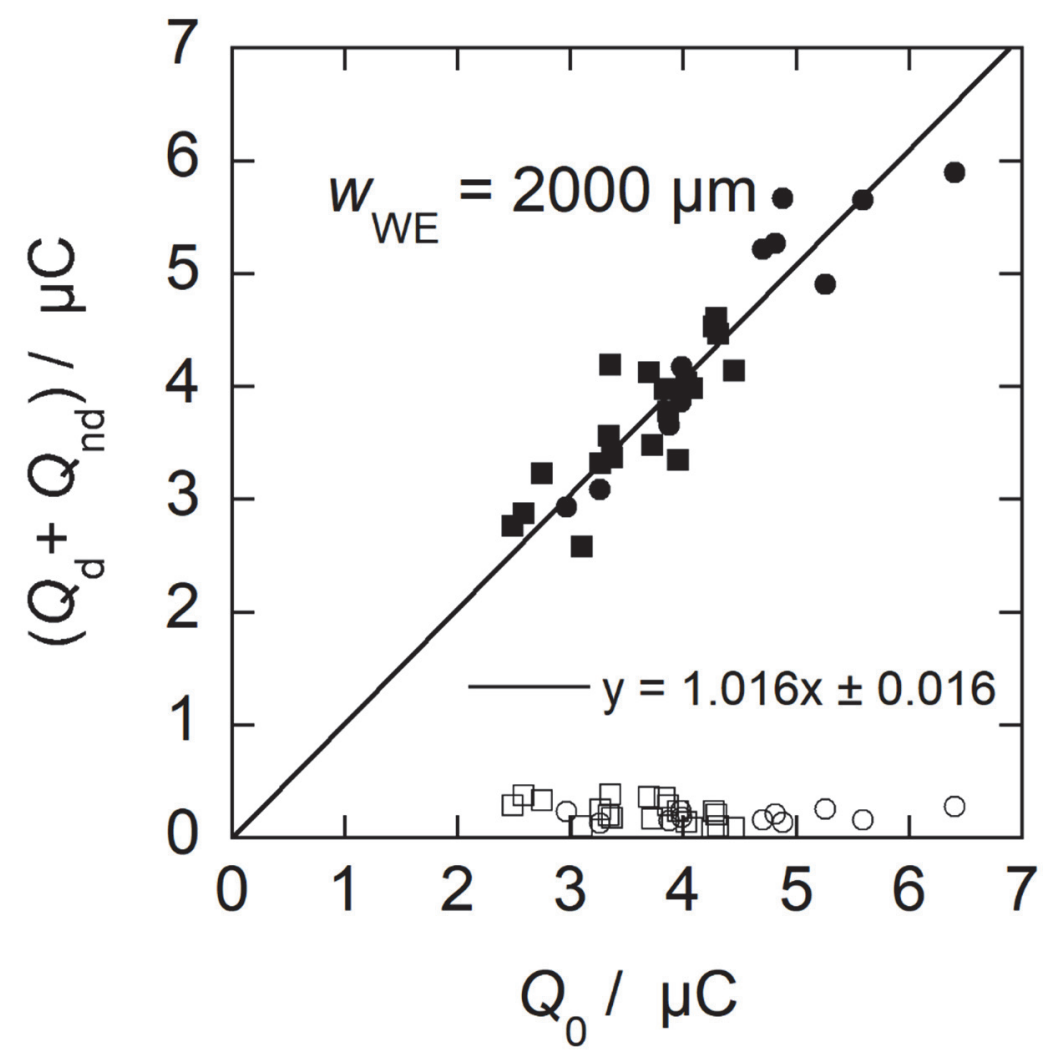

Fig. 8. Total charge quantity $\left(Q_{\mathrm{d}+} Q_{\mathrm{nd}}\right)$ as a function of the charge quantity $Q_{0}$ for $w_{\mathrm{WE}}=2000 \mu \mathrm{m}$ : Linear curve fits (solid lines) of $\left(Q_{\mathrm{d}+} Q_{\mathrm{nd}}\right)$ (solid symbols), and corresponding values of $Q_{\text {nd }}$ (open symbols) calculated from Eq. (8). 


\section{Graphical abstract}

Circulating droplets in microfluidic channels can be quantitatively electrolyzed or analyzed using kchannels.
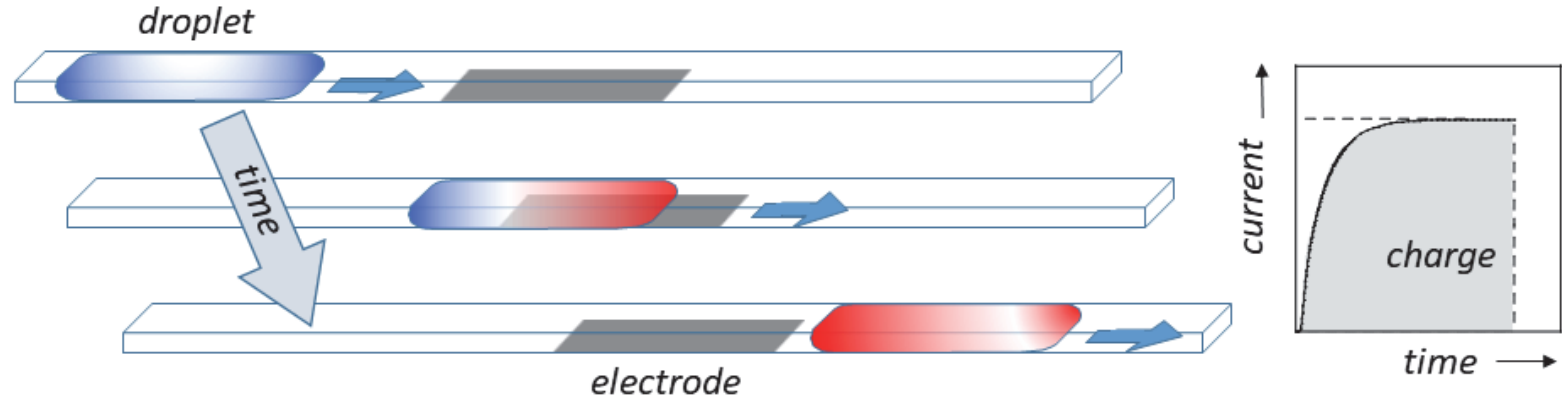

0

1

$c / c_{0}$ 


\section{Highlights}

- Droplets can be quantitatively electrolyzed without influence of the counter electrode

- The velocity and size of the droplets do not need to be assessed

- No calibration is required to analyze or convert the droplet content

- The geometry of the microdevice is optimized for a wide range of droplet properties 